\title{
Catheter Ablation of Atriofascicular Mahaim Fibers Guided by the Activation Potential
}

\author{
Márcio Augusto Silva, Gel Berardi, Alessandro Kraemer, Elenir Nadalin, José Carlos Moura Jorge \\ Curitiba, PR - Brazil
}

\begin{abstract}
Objective - To determine whether recording of the activation potential may be used as an isolated criterion to guide catheter ablation of atriofascicular Mahaim fibers.

Methods - We studied 6 patients (5 females, mean age of $26 \pm 7.3$ years) with paroxysmal tachycardias with a wide QRS complex, whose electrophysiological study diagnosed atriofascicular Mahaim fibers. Mapping and catheter ablation were performed in sinus rhythm, guided only by the recording of the activation potential of the fiber.
\end{abstract}

Results - Efficacy in ablation was achieved in all patients. The fibers were located in the right lateral region of the tricuspid ring in 3 patients, right posterolateral region in 2, and right anterolateral region in $1 . A$ mean of $5.3 \pm 3$ radiofrequency applications was performed. The mean fluoroscopy time was $46.6 \pm 25$ minutes, and the mean duration of the procedure was $178.6 \pm 108$ minutes. No complication occurred. In a mean 20-month followup, all patients were asymptomatic and receiving no antiarrhythmic drugs.

Conclusion - Catheter ablation of Mahaim fibers may be performed with good safety and efficacy by mapping the activation potential of the tricuspid ring in sinus rhythm.

Keywords: Mahaim fibers, catheter ablation, atriofascicular accessory pathways

Hospital Universitário Cajuru - PUC/PR

Mailing address: Márcio Augusto Silva - Rua Saint Hilaire, 122/1103 - 80240140 - Curitiba, PR, Brazil - E-mail: masilva@sulbbs.com.br

English version by Stela Maris C. e Gandour
In 1938, Mahaim et al ${ }^{1}$ described some nodoventricular conduction fibers, which formed the substrate for a special type of preexcitation syndrome characterized by little or no preexcitation on the basal electrocardiogram and tachycardias with left bundle-branch block morphology. Later studies ${ }^{2-6}$ showed that these fibers had special properties, such as exclusive anterograde and decremental conduction, and could be located in sites distant from the atrioventricular node and eliminated by surgery or catheter ablation. Recently, most of these pathways have been classified as atriofascicular fibers that connect the right atrium to distal portions of the right branch of the His bundle with decremental properties ${ }^{7,8}$.

Because they have different anatomical and electrophysiological characteristics, the following special ablation techniques have been considered: 1) atrial stimulation (pace-mapping) along the tricuspid ring, looking for the shortest "spike-Delta" interval" ; 2) mapping with extrastimuli during antidromic tachycardia, searching for the point of greatest "preexcitation" of the subsequent QRS; and 3) mapping of the activation potential of the fiber in sinus rhythm ${ }^{10-13}$. The latter has been considered more precise than the others; however, due to the rarity of this type of fibers, little experience has been reported in the medical literature.

This study analyzed the results of radiofrequency catheter ablation of Mahaim fibers in 6 consecutive patients by using the exclusive mapping of fiber activation potential.

\section{Methods}

From February 1999 to February 2000, 101 patients with accessory pathways underwent ablation at our institution, 6 (5.94\%) of whom met the electrophysiological criteria for tachycardia mediated by Mahaim fibers. All patients were referred to our service because of tachycardia crises with a wide QRS complex and left bundle-branch block morphology difficult to control clinically. These patients were taking amiodarone at the time of the indication of the electrophysiological study. Five patients were females and their 
mean age was $26.5 \pm 7.3$ years (ranging from 16 to 36 years). One patient had mild aortic insufficiency with no hemodynamic repercussions, and the remaining had no structural heart disease. No patient had ventricular preexcitation on the basal electrocardiogram.

The procedure was performed after an 8-hour fasting period, with written formal consent provided by the patient or his guardian, previous suspension of amiodarone for 1 month, and sedated with intravenous midazolam and fentanyl. Two 7Fr quadripolar catheters were introduced through puncture of the right femoral vein, and, guided by fluoroscopy, positioned in the region of the high right atrium and His bundle; for recording the ventricular electrogram and ventricular stimulation, 1 of these catheters was introduced into the right ventricle. The 4.2 version of the PCEMS system (Personal Computer Electrophysiology Measurement System; University of Limburg, The Netherlands) was used with frequency cut between 50 and $500 \mathrm{~Hz}$ and a velocity of up to $300 \mathrm{~mm} / \mathrm{s}$ to record the intracavitary signals and peripheral electrocardiogram and also to programmed stimulation.

The criteria for the diagnosis of Mahaim fiber were as follows: absence of ventricular preexcitation in sinus rhythm or discrete preexcitation with left bundle-branch block morphology; presence of progressive ventricular preexcitation during continuous atrial stimulation associated with an increase in the A-V interval and a reduction in the $\mathrm{H}-\mathrm{V}$ interval; induction of antidromic atrioventricular tachycardia with left bundle-branch block morphology, this morphology being similar to that of maximum preexcitation ${ }^{9,10,11-16}$. Bardy et al ${ }^{17}$ described typical Mahaim tachycardia as follows: 1) left bundle-branch block morphology; 2) QRS axis superior to or between $0^{\circ}$ and $75^{\circ}$; 3 ) presence of $\mathrm{R}$ wave in $\mathrm{D} 1$; 4) presence of $\mathrm{rS}$ in $\mathrm{V} 1$; and 5) QRS transition in $\mathrm{V} 4$ or after V4.

The distal insertion of the Mahaim fiber was defined by the earliest recording of the bipolar ventricular electrogram in regard to the beginning of the QRS complex in tachycardia or during atrial stimulation. The insertion was considered fascicular (atriofascicular fiber) when the earliest ventricular activation was recorded in the right ventricular apical region, close to the distal portion of the right bundlebranch, and the insertion was considered ventricular (atrioventricular fiber) when the earliest activation was recorded close to the atrioventricular ring ${ }^{14,16}$.

Four-mm 7Fr catheters with deflectable tip (EP Technologies) and radiofrequency generator with temperature control (EP Technologies) were used for ablation. Long introducers (Swartz TM Guiding Introducers, SR3 and SR4, DAIG, St. Jude Medical Company) were used in some procedures to better stabilize the catheter.

Recording of the activation potential of the Mahaim fiber for ablation in sinus rhythm was sought during mapping of the tricuspid ring. The radiofrequency applications were performed with a power limit of $50 \mathrm{w}$ and temperature of $70^{\circ}$ Celsius for 60 seconds. After each application, a new programmed atrial stimulation was performed. Success of the procedure was defined as the absence of ventricular preexcitation during atrial stimulation and no tachycardia induction according to the same protocol of stimulation of the beginning of the procedure. After 20 minutes, programmed stimulation was repeated with and without intravenous infusion of isoproterenol $(1-2 \mathrm{mg} / \mathrm{min})$.

\section{Results}

Six patients met the criteria for the diagnosis of Mahaim fiber. During programmed continuous atrial stimulation with progressively shorter coupling intervals, an increase in the $\mathrm{A}-\mathrm{V}$ and $\mathrm{A}-\mathrm{H}$ intervals and a reduction in the $\mathrm{H}-\mathrm{V}$ interval were observed concomitantly with progressive ventricular preexcitation with left bundle-branch block morphology (fig. 1).

Recording of the earliest ventricular electrogram during tachycardia and maximum ventricular preexcitation was obtained in the apical region of the right ventricle, close to the distal portions of the right bundle-branch and distant from the $\mathrm{AV}$ ring, characterizing these fibers as atriofascicular in all patients (fig. 2).

Antidromic AV tachycardia with left bundle-branch block morphology (QRS with the same morphology observed during maximal preexcitation) and a mean cycle of $333.6 \pm 32 \mathrm{~ms}$ were induced in all patients (fig. 3 ). The retrograde conduction observed during tachycardia, as well as that observed during ventricular stimulation, was characterized as nodal in 5 patients, and as occult right posteroseptal (RPS) AV in 1 patient (P6). No retrograde conduction of the Mahaim fiber was observed. No sign of a double nodal pathway was observed in any patient.

Mapping was performed in sinus rhythm in all patients. The site of recording of the activation potential of the Mahaim fiber in the tricuspid ring was chosen for ablation in all patients (fig. 4). Ablation was successful in all patients.

Three patients (P1, P2, P6) required 2 sessions of ablation. In patients $\mathrm{P} 1$ and $\mathrm{P} 2$, mechanical conduction block (catheter touch) in the Mahaim fiber during mapping in the first procedure occurred with disappearance of ventricular preexcitation on atrial stimulation and of the activation potential, making ablation impossible. These patients underwent a new successful session after 1 and 2 months, respectively. Patient P6 had supraventricular tachycardia (narrow QRS) after a 4-month follow-up and underwent a new procedure, with recurrence of the occult AV (RPS), which was eliminated again with radiofrequency (no sign of Mahaim fiber conduction was observed in the second session). After the first 2 procedures in patients $\mathrm{P} 1$ and $\mathrm{P} 2$, we began to use the long introducers (SR3 and SR4), which provided better stabilization of the ablation catheter.

The Mahaim fiber was located in the right lateral region in 3 patients, in the right posterolateral region in 2 patients, and in the right anterolateral region in 1 patient. A mean of $5.3 \pm 3$ radiofrequency applications per patient was performed (2 - 11), with a fluoroscopy time of $46.6 \pm 25 \mathrm{minu}-$ tes (26-82), and total time of the procedure of $178.6 \pm 108 \mathrm{mi}-$ nutes $(95-340)$. No complications occurred (tab. I). 


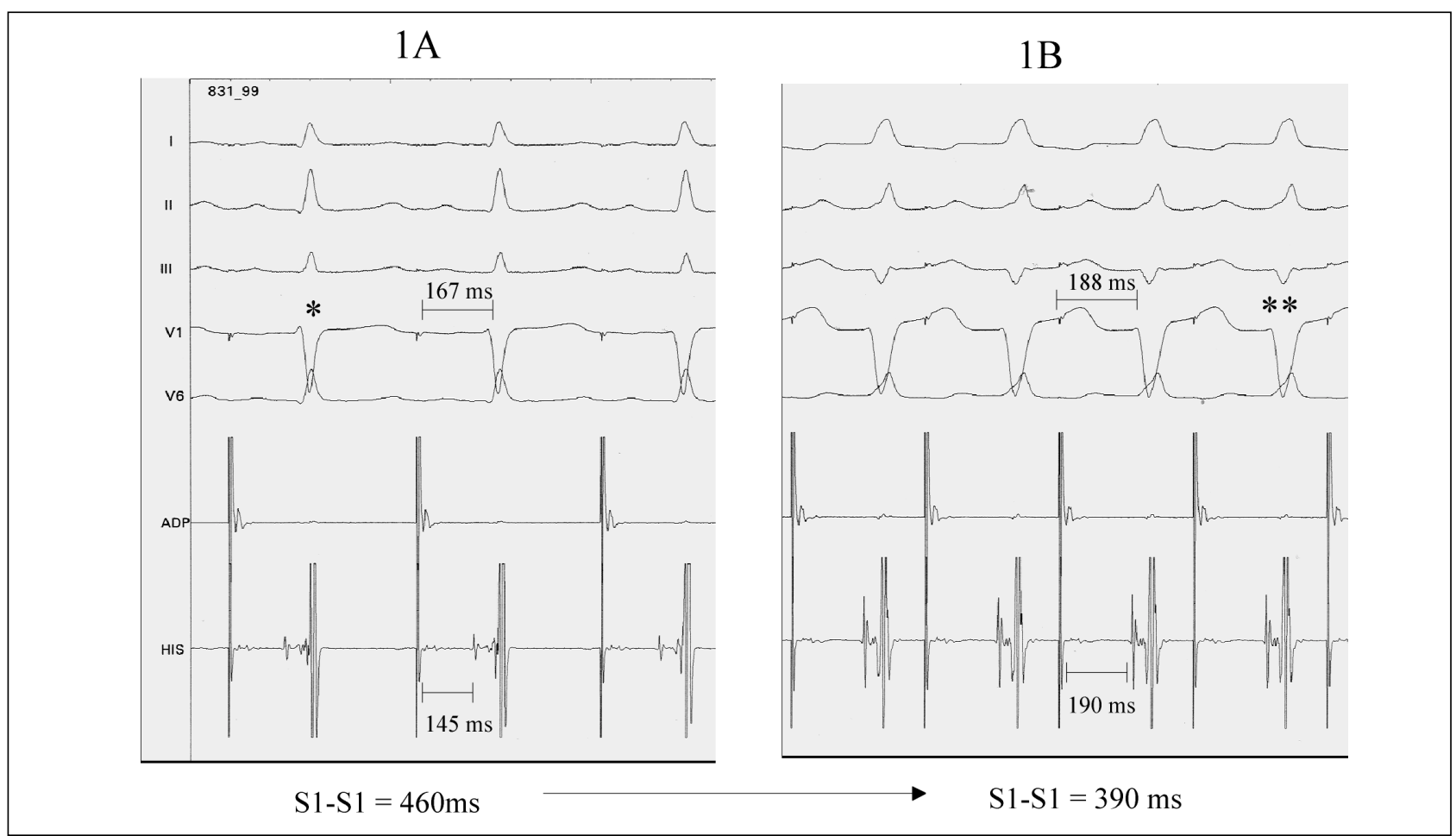

Fig. 1 - Continuous programmed stimulation in high right atrium showing: (1A) with a stimulation cycle (S1-S1) of $460 \mathrm{~ms}$, mild ventricular preexcitation occurs $(*) ;(1 \mathrm{~B})$ continuing the tracing, progressive ventricular preexcitation is observed during the stimulation at progressively shorter cycles $(\mathrm{S} 1-\mathrm{S} 1=390 \mathrm{~ms})(* *)$ with extension of the $\mathrm{A}-\mathrm{H}$ and A-V intervals.

The patients are asymptomatic, with no antiarrhythmic drugs, in a mean $20 \pm 3.5$-month follow-up.

\section{Discussion}

We report the cases of 6 patients $(5.9 \%$ of the patients with ventriculoatrial in the same period) who had tachycardia crises with wide QRS and left bundle-branch block morphology, and who met the diagnostic criteria for atriofascicular Mahaim fibers on electrophysiological study. No patient had ventricular preexcitation in sinus rhythm, but, during continuous atrial stimulation, progressive ventricular preexcitation was observed with left bundle-branch block morphology associated with an increase in the $\mathrm{A}-\mathrm{V}$ interval,

\begin{tabular}{|cccccccc|}
\hline \multicolumn{7}{|c|}{ Table I-Characteristics of the patients } \\
\hline & Sex & Age & Session & $\begin{array}{c}\text { Fiber } \\
\text { site }\end{array}$ & $\begin{array}{c}\text { N }^{\circ} \text { of } \\
\text { applications }\end{array}$ & $\begin{array}{c}\text { Total } \\
\text { (min) }\end{array}$ & $\begin{array}{c}\text { Time of fluo- } \\
\text { roscopy (min) }\end{array}$ \\
\hline P1 & F & 24 & 1 & RL & 0 & 200 & 50 \\
P2 & M & 16 & 1 & RPL & 3 & 125 & 34 \\
P3 & F & 36 & 1 & RL & 6 & 105 & 26 \\
P4 & F & 34 & 1 & RAL & 4 & 95 & 26 \\
P5 & F & 24 & 1 & RPK & 2 & 127 & 36 \\
P6 & F & 25 & 1 & RL* & 4 & 110 & 34 \\
\hline \multicolumn{7}{|l}{ P1 - P6 - patients; RL- right lateral; RPL- right posterolateral; RAL- right } \\
anterolateral; * with atrioventricular accessory occult right posteroseptal \\
pathway associated with Mahaim fiber. \\
\hline
\end{tabular}

a reduction in the $\mathrm{H}-\mathrm{V}$ interval, and induction of antidromic atrioventricular tachycardia with left bundle-branch block morphology similar to that of ventricular preexcitation. Radiofrequency ablation was performed at the site where the activation potential of the Mahaim fiber in sinus rhythm was recorded, with a final rate of success of $100 \%$ and no recurrences or complications.

Mahaim fibers correspond approximately to 3\% of all accessory pathways. The therapeutic options include

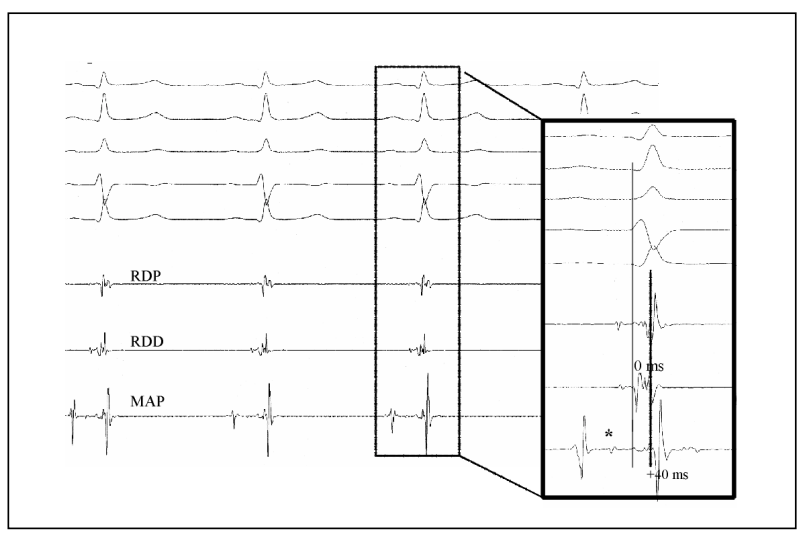

Fig. 2 - Simultaneous recording of the Mahaim (*) and right bundle-branch potentials in sinus rhythm. In the highlighted tracing, the ventricular electrogram is "earlier" in regard to the QRS complex in the region of the distal right bundle branch (DRBB) as compared with the tricuspid ring (MAP) in the region of atrial insertion of the Mahaim fiber $(0 \mathrm{~ms} v+40 \mathrm{~ms})$. This finding confirms that the ventricular insertion of the fiber relates to the distal portion of the right bundle branch characterizing it as atriofascicular. 


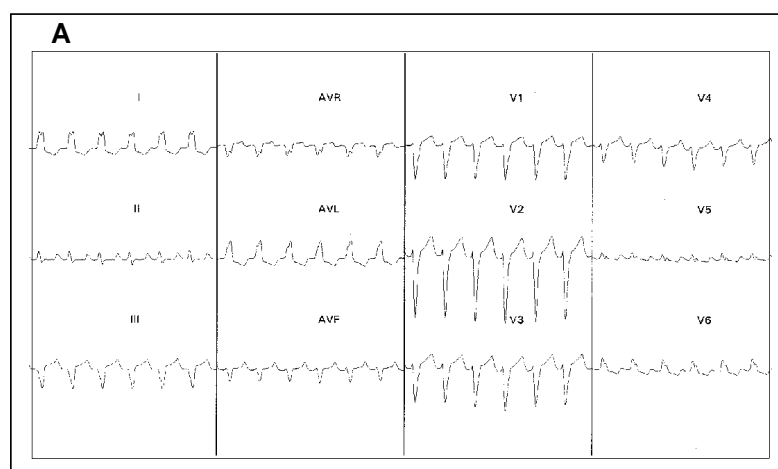

B

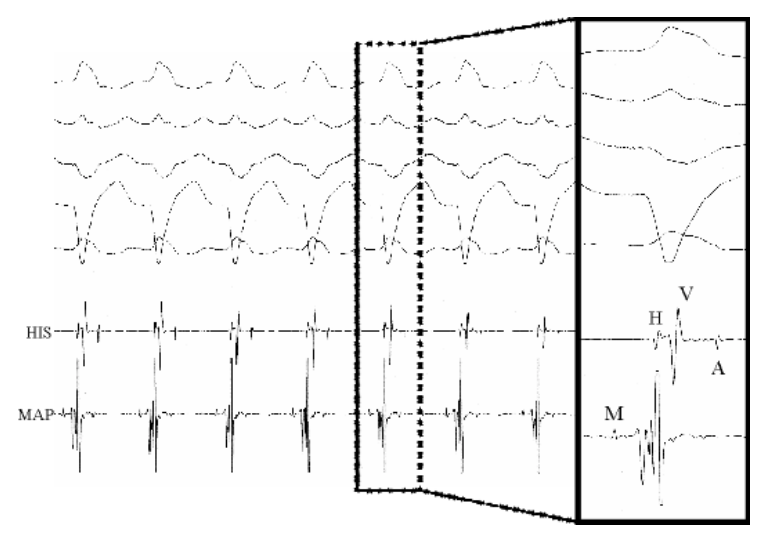

Fig. 3 - Mahaim antidromic tachycardia. (A) 12-lead electrocardiographic tracing of the Mahaim tachycardia; (B) endocavitary recordings during tachycardia with catheters positioned in the His bundle region (HIS) and upon the Mahaim fiber in the tricuspid ring (MAP). In the highlighted tracing, the anterograde component of the circuit is provided by the Mahaim fiber and the retrograde component by the His bundle. H - His potential; V- ventricular electrogram; A- atrial electrogram; MMahaim potential.

pharmacological treatment, catheter ablation ${ }^{5,6,9-12,18}$, and surgery ${ }^{7,12}$. The first experiences with catheter ablation used high-energy shocks in the atrioventricular node region. In these cases, the tachycardias were eliminated, but the patients had complete $\mathrm{QRS}$ preexcitation with a long A$\mathrm{V}$ interval and decremental properties ${ }^{5,6}$. These findings contributed to the hypothesis that the Mahaim fibers and the atrioventricular node were anatomically distant, contrary to that which had been previously reported. Haissaguerre et al ${ }^{19}$ showed that ablation of the right atriofascicular fibers with high-energy shocks in the right ventricular apex at a point proximal to the insertion of the fiber in the right ventricle or in the right bundle-branch was possible, preserving the conduction system. However, this could have proarrhythmic effects, because of the increase in the time of ventriculoatrial conduction, making the tachycardia crises more frequent ${ }^{10,14}$.

With the evolution of electrophysiology, more satisfactory results were obtained, aiming at the ablation of the atrial insertion of these fibers ${ }^{9-13}$. Because its distal insertion is not located adjacent to the tricuspid ring, as with the accessory atrioventricular pathways (Wolff-ParkinsonWhite syndrome), mapping the earliest ventricular activation in regard to QRS is useless in these cases. In addition,

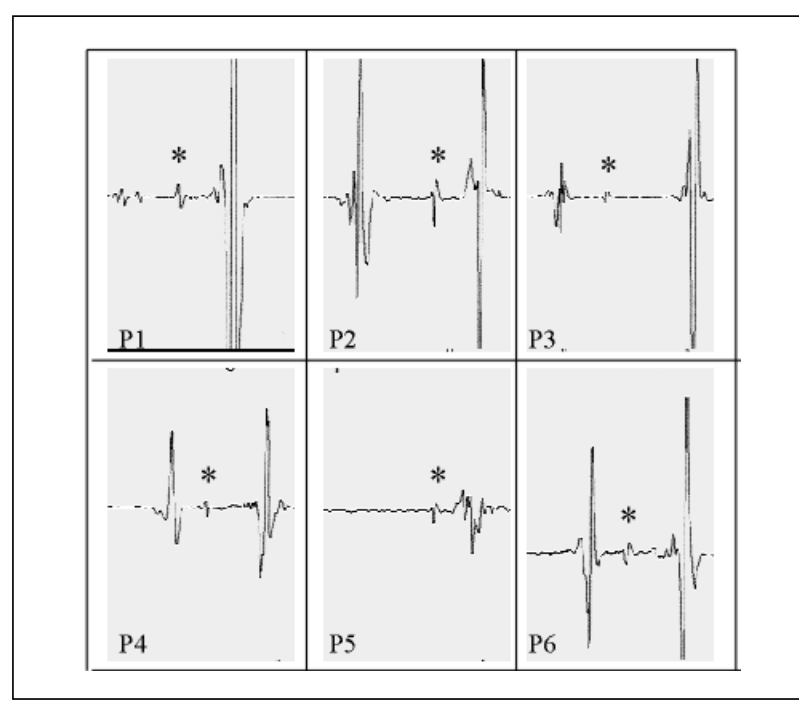

Fig. 4-Activation potential of the Mahaim fiber $\left({ }^{*}\right)$-recordings at the ablation sites of each patient ( $\mathrm{P} 1$ to $\mathrm{P} 6$ ).

because these fibers do not have retrograde conduction, mapping the ventriculoatrial conduction is not possible. Therefore, the following special techniques for mapping these fibers were reported: 1) mapping atrial extrastimuli along the tricuspid ring to identify the shortest spike-Delta interval (pace-mapping);2) release of extrastimuli along the tricuspid ring during antidromic tachycardia searching for the point of the greatest "advancement" (preexcitation) of the subsequent QRS; and 3) mapping the fiber activation potential in sinus rhythm ${ }^{9-14}$.

Klein et al ${ }^{9}$ reported on 4 patients who underwent ablation with pace-mapping in the atrium. Three patients had atriofascicular fibers and 1 patient had atrioventricular fibers. Ablation was successful in all patients, with a mean of 15 radiofrequency applications. The elevated number of applications reported in this and other series ${ }^{13}$ suggests that the technique, although effective, may be imprecise in locating the exact atrial insertion of the fiber. On the other hand, the release of extrastimuli in tachycardia has been considered more technically difficult and time-consuming, and no consistent results have been reported in the medical literature so far $^{9,14}$.

Mapping the fiber using its activation potential in sinus rhythm has been reported as a more accurate technique $^{10-13}$. These potentials (activation potential) are similar to the His potential and may be recorded mainly in the lateral and anterolateral regions of the tricuspid ring ${ }^{10}$. Okishige et al ${ }^{13}$ have reported that Mahaim fibers have 2 portions: a proximal portion with decremental properties, which generates a slow potential hardly recorded during mapping, and a distal portion with nondecremental conduction properties ("everything-or-nothing"), similar to the Kent bundle, which generates a fast potential (spiked potential). According to the authors, the best method to eliminate these fibers is to apply radiofrequency at the recording site of the fast potential.

McClelland et al ${ }^{10}$ reported the first great results with 
this technique. Of the 26 patients with Mahaim fibers, 23 had atriofascicular fibers. Catheter ablation guided by the activation potential could be performed in 22 patients, and, in 1 , mapping was performed with atrial stimulation because of failure in recording the activation potential. They achieved a final success rate of $100 \%$. In this series, the applications were performed during atrial stimulation or tachycardia, to observe the conduction block in the fiber. To improve catheter stability, right subclavian vein access was used in 15 patients.

Grogin et al ${ }^{11}$ obtained successful ablation using this technique in 4 of 4 patients with atriofascicular fibers, with a mean of 10 radiofrequency applications. Similar results were reported by Brugada et al ${ }^{12}$, with a mean of 7 radiofrequency applications in 4 consecutive patients.

Some authors, however, associate mapping forms as follows: using atrial stimulation on the atrial surface of the tricuspid ring, the site of the smallest spike-Delta is determined, and, in this region, a careful search for the activation potential of the fiber in sinus rhythm is carried out ${ }^{13,14}$.

Cappato et al ${ }^{18}$ reported on a mapping technique, in which mechanical conduction block of the Mahaim fiber was attempted by manipulating the catheter, and applying radiofrequency in this site. As already reported, conduction block in 1 Mahaim fiber due to mechanical trauma is accompanied by disappearance of its activation potential, and conduction through the fiber may not return during the same procedure ${ }^{10}$. In case the ablation catheter is not stable at the site of block, the application may not be accurate, leading to doubtful results. This happened with the first 2 patients in our series after mechanical block, in which no activation potential of the fibers could be recorded, leading to a second ablation session. After these first cases, we began to routinely use long introducers with premolded curves for the right lateral and posterolateral pathways (SR3 e SR4), assuring greater catheter stability.

In conclusion, mapping of the tricuspid ring in sinus rhythm to record the activation potential of Mahaim fibers may be used as the only criterion for catheter ablation of these fibers, and it has proved to be an effective and safe method.

\section{References}

1. Mahaim I, Benatt A. Nouvelles recherches sur les connections supérieures de la branche du faisceau de His-Tawara avec cloison interventriculaire. Cardiologia 1937; 1: 61-76.

2. Wellens HJJ. Tachycardias related to the preexcitation syndrome. In: Wellens HJ, ed. Electrical Stimulation of the Heart in the Study and Treatment of Tachycardias. Baltimore: University Park Press, 1971: 97-109.

3. Anderson BH, Becker AE, Brechenmacher C, et al. Ventricular pre-excitation: a proposed nomenclature for its substrates. Eur J Cardiol 1975; 3: 27-36.

4. Gillete PC, Garson A Jr, Cooley DA, McNamara DG. Prolonged and decremental antegrade conduction properties in right anterior acessory conections: wide QRS antidromic tachycardia of left bundle branch block pattern withoutWolff-Parkinson-White configurationin sinus rhythm. Am Heart J 1982; 103: 66-74.

5. Bhandari A, Morady F, Shen EN, et al. Cateter induced His bundle ablation in a patient with reentrant tachycardia associated with a nodoventricular tract. J Am Coll Cardiol 1984; 4: 611-6.

6. Ellenboghen KA, O'Callaghan WG, Colavita PG, et al. Catheter atrioventricular junction ablation for recurrent supraventricular tachycardias with nodoventricular fibers. Am J Cardiol 1985; 55: 1227-9.

7. Klein GJ, Guiraudon GM, Kerr CR, et al. Nodoventricular accessory pathway: evidence for a distinct accessory atrioventricular pathway with atrioventricular node-like properties. J Am Coll Cardiol 1988; 11: 1035-40.

8. Tchou PT, Lehmann MH, Jazayeri M, Akhtar M. Atriofascicular connection or a nodoventricular Mahaim fiber? Electrophysiologic elucidation of the pathway and associated reentrant circuit. Circulation 1988; 77: 837-48.

9. Klein L, Hackett FK, Zipes DP, Miles WM. Radiofrequency catheter ablation of Mahaim fibers at the tricuspid annulus. Circulation 1993; 87: 738-47.

10. McClelland JH, Xunzhang W, Beckman KJ, et al. Radiofrequency catheter abla- tion of right atriofascicular (Mahaim) accessory pathways guided by accessory pathway activation potentials. Circulation 1994; 89: 2655-66.

11. Grogin HR, Lee RJ, Kwasman M, et al. Radiofrequency catheter ablation of atriofascicular and nodoventricular Mahaim tracts. Circulation 1994; 90: 272-81.

12. Brugada J, Martínez-Sánchez J, Kuzmicic B, etal. Radiofrequency catheter ablation of atriofascicular accessory pathways guided by discrete electrical potentials recorded at the tricuspid annulus". Pacing Clin Electrophysiol 1995; 18: 1388-94.

13. Okishige K, Goseki Y, Itoh A, et al. New electrophysiologic features and catheter ablation of atrioventricular and atriofascicular accessory pathways: evidence of decremental conduction and the anatomic structure of the Mahaim pathway. $\mathrm{J}$ Cardiovasc Electrophysiol 1998; 9: 22-33.

14. Zhu DWX. Ablation of right free wall and Mahaim accessory pathways. In: Singer I, Barold SS, Camm AJ, eds. Nonpharmacological Therapy of Arrhytmias for the 21st Century. The State of the Art. New York: Futura Publishing Company, 1998: 89-116.

15. Aliot E, Chillou C, D'Allones GR, Mabo P, Sadoul N. Mahaim tachycardias. Eur Heart J 1998; 19(suppl E): E25-E31.

16. Miles WM, Klein LS, Rardon DP, Mitrani RD, Zipes DP. Atrioventricular reentry and variants: mechanisms, clinical features, and management. In: Zipes \& Jalife, eds. Cardiac Electrophysiology: from Cell to Bedside. $2^{\text {nd }}$ edition. Philadelphia: WB Saunders, 1995: 638-55.

17. Bardy GH, Fedor JM, German LD, et al. Surface eletrocardiographic clues suggesting presence of a nodo-fascicular Mahaim fiber. J Am Coll Cardiol 1984; 3: 1161

18. Capatto R, Schluter M, Weib C, et al. Catheter induced mechanical conduction block of right-sided accessory fibers with Mahaim-type preexcitation to guide radiofrequency ablation. Circulation 1994; 90: 282-90.

19. Haissaguerre M, Warin JF, Metayer P, etal. Catheter ablation of Mahaim fibers with preservation of atrioventricular nodal conduction. Circulation 1990; 82: 418-27. 